\title{
Adaptive Synchronization Design for Chaotic Systems via a Scalar Driving Signal
}

\author{
Kuang-Yow Lian, Member, IEEE, Peter Liu, Student Member, IEEE, Tung-Sheng Chiang, and Chian-Song Chiu
}

\begin{abstract}
Using a scalar driving signal, synchronization for a class of chaotic systems has been developed in this study. For chaotic systems characterized by nonlinearity, which depends only on the available output, a unified approach is developed by carefully extending the conventional adaptive observer design. For exactly known chaotic systems, an exponential convergence of synchronization is achieved in the large. When mismatched parameters are presented, this method performs the asymptotic synchronization of output state in the large. The convergence of the estimated parameter error is related to an implicit condition of persistent excitation (PE) on internal signals. From the broad spectrum characteristics of the chaotic driving signal, we reformulate the implicit PE condition as an condition on injection inputs. If this condition is satisfied, the estimated parameters converge to true values and exponential synchronization of all internal states is guaranteed. Two typical examples, including Duffing-Holmes system and Chua's circuit, are considered as illustrations to demonstrate the effectiveness of the adaptive synchronizer. Furthermore, the robustness of adaptive synchronization in presence of measurement noise is considered where the update law is modified. Finally, numerical simulations and DSP-based experiments show the validity of theoretical derivations.
\end{abstract}

Index Terms-Adaptive observer, chaotic synchronization, persistent excitation.

\section{INTRODUCTION}

C HAOTIC systems have been studied and known to exhibit complex dynamical behavior in past two decades. The interest in chaotic systems lies mostly upon their complex, unpredictable behavior, and extreme sensitivity to initial conditions as well as parameter variation. These properties in turn, are the ones most commonly characterized as their prominent features. In light of a broad-spectrum and noise-like chaotic signal, many theories of control and synchronization have been developed.

According to the synthesis method proposed in [1], chaotic synchronization is where two chaotic systems with suitable coupling produce identical oscillations after a transient time even when starting from arbitrary initial conditions. In many works [2]-[18], different theories have been proposed to achieve synchronization with exactly known or uncertain conditions. From the observation of coupling configurations [2], the current synchronization structures are classified into: 1) master-slave

Manuscript received January 24, 2000; revised June 22, 2001. This work was supported by the National Science Council, R.O.C., under Grant NSC-89-2213E033-006. This paper was recommended by Associate Editor M. J. Ogorzalek.

K.-Y. Lian, P. Liu and C.-S. Chiu are with the Department of Electrical Engineering, Chung-Yuan Christian University, Chung-Li 32023, Taiwan, R.O.C. (e-mail: lian@dec.ee.cycu.edu.tw).

T.-S. Chiang is with the Department of Electrical Engineering, Ching-Yun Institute of Technology, Chung-Li 320, Taiwan, R.O.C.

Publisher Item Identifier S 1057-7122(02)00281-7. synchronization [3]-[10] and 2) mutual synchronization [2]. The former has unidirectional coupling, while the latter is with bidirectional coupling. Most theoretical frameworks and stability analysis for synchronization have stemmed from the master-slave configuration. This master-slave configuration consists of the original chaotic system as a drive system to provide a coupling signal to drive another system called the response system to synchrony. Several control approaches, including model reference control and observer design, are widely used for synchronization. For model reference control approach (or state feedback control), the states of the response system converge to the drive system by available inputs (driving signals) [12]-[14]. The drawback of this technique is that the full states of the drive system are usually required in implementation. On the other hand, a response system plays the role of a observer to reconstruct or to estimate the dynamics of the given chaotic drive system via an available output. The main feature of observer-based concepts, employing linear-observer or nonlinear-observer design, is that it is systematic. However, most of these schemes, when concerned with the region of convergence, are at best arbitrarily large by using high gains from assuming Lipschitz conditions of nonlinear terms [6]-[8], or transmitting the nonlinear terms [9], [10]. For more advanced works, adaptive synchronization [11]-[18] are introduced to present not only nonidentical initial conditions but also parameter mismatch between master and slave systems. A Lyapunov based approach [15], [16], using the LMS adaptive algorithm, is proposed to solve the adaptive synchronization of general chaotic systems. Although adaptive synchronization can be developed for master-slave and mutual configurations, the adaptive mechanism is implemented by full-state feedback for full parameter mismatch. To overcome this problem, the speed-gradient method [17] has been successfully applied to synchronization by using an approximate mean-value compensation of states. For the mutual coupling structure, a passification approach [18] is designed by utilizing output feedback. Several works ensure only semi-global convergence due to assuming Lipschitz conditions on the nonlinear terms.

Inspired by works [15]-[18], an adaptive observer-based approach is proposed for a class of chaotic systems in this paper. The main results of this work are that synchronization and adaptive synchronization without Lipschitz conditions can be solved simultaneously by using a scalar driving signal. If the chaotic signal is sufficiently rich, namely persistent excitation (PE) condition is satisfied, estimated parameters converge to true values and the exponential synchronization of complete states are guaranteed. The PE condition can be satisfied easier if the transmitted signal is a chaotic signal. This is reasonable 
since chaotic systems are broadband, noise-like and have a rich dynamical pattern. The proposed framework allows one to solve the problem for a class of chaotic systems characterized by the nonlinear terms of the drive system depending only on the available output. This is valid even after an appropriate change of coordinates. From the viewpoint that the nonlinear terms are injection inputs in the dynamics of a drive system, the input-output equivalent representation is easily obtained. Then a unified framework is devoted to synchronization and adaptive synchronization by carefully extending the adaptive observer scheme [19]. Furthermore, the robustness of the proposed adaptive synchronization is established with the converse Lyapunov method. To further show the validity of theoretical derivations, numerical simulations and DSP-based experiments are carried out on the Chua's circuit.

The paper is organized as follows. In Section II, a unified design method for synchronization and adaptive synchronization is presented. Two examples of typical chaotic systems, including Duffing equations and Chua's circuit, are illustrated in Section III. Also, the effectiveness of the proposed adaptive scheme is demonstrated by numerical simulations. For the robustness issue with respect to measurement noise, a modified adaptive law is suggested in Section IV. Some oscilloscope images of DSP-based experiments are shown to illustrate the consistency of the theoretical results with practical implementations in Section V. Finally, some conclusions are made in Section VI.

\section{SYNCHRONIZATION AND ADAPTIVE SYNCHRONIZATION}

\section{A. Model Representation}

For a class of chaotic systems in which the nonlinearity depends only on the available output, we introduce an approach for chaotic synchronization using a scalar driving signal. The general output injection form for these systems is described by

$$
\begin{aligned}
\sum_{1}: \quad \dot{x}(t) & =A x(t)+\sum_{i=1}^{p} B_{i} f_{i}(y(t), t) \\
y & =C x(t)
\end{aligned}
$$

where $x \in R^{n}$ represents the state vector; the system output $y \in R$ denotes the driving signal; the injection inputs $f_{i} \in R$, $i=1, \ldots, p$, may denote the driving signal, the nonlinearity in the system dynamics, the oscillated force, dc bias or a combination of them; and $A, B_{i}, C$ are constant matrices with appropriate dimensions. Some examples of this class of nonlinear systems include the Duffing-Holmes equation, Van der Pol oscillator, Chua's circuit and Rossler system, etc. Furthermore, we assume the matrix pair $(A, C)$ is observable and $\left(A, B_{i}\right)$ $\forall i=1, \ldots, p$ are controllable. These properties are satisfied when we rewrite the mentioned chaotic systems into the form (1).

The synchronization for this category of chaotic systems is based on the linear observer design derived using the output signal $y$ to drive the response system. This technique, presented in [7], [8], needs the Lipschitz condition on nonlinear terms $f_{i}$, which results in the convergence achieved by high observer gains. To overcome this problem, we introduce an observerbased approach to chaotic synchronization. In preparation for the synchronizer design, a property of system $\sum_{1}$ is discussed as follows:

Proposition 1: System $\sum_{1}$ is input-output equivalent to a $[(p+1)(n-1)+1]$ dimensional system

$$
\begin{aligned}
\dot{z}_{1}(t) & =-r z_{1}(t)+\vartheta^{\mathrm{T}} f(y, t)+\theta^{\mathrm{T}} \omega(t) \\
\dot{\omega}_{i}(t) & =\Lambda \omega_{i}(t)+B_{\lambda} f_{i}(y, t), \quad i=1, \ldots, p+1 \\
y(t) & =z_{1}(t)
\end{aligned}
$$

where $f=\operatorname{col}\left[f_{1}, f_{2}, \ldots, f_{p+1}\right] ; \omega(t)=\operatorname{col}\left[\omega_{1}, \omega_{2}, \ldots\right.$, $\left.\omega_{p+1}\right] ; \Lambda \in R^{(n-1) \times(n-1)}$ and $B_{\lambda} \in R^{n-1}$ are constant matrices with appropriate dimensions. Furthermore, the original states $x$ can be expressed in terms of the new states $z=(z, \omega)$ as $x=F z$ for a constant matrix $F \in R^{n \times[(p+1)(n-1)+1]}$.

Proof: The terms $f_{i}(y(t), t)$ arising from the output injection can be regarded as inputs to system $\sum_{1}$. From this viewpoint, $\sum_{1}$ is described by the following strictly proper transfer functions:

$$
y=\sum_{i=1}^{p} C(s I-A)^{-1} B_{i}\left[f_{i}\right]=\sum_{i=1}^{p} \frac{n_{i}(s)}{d(s)}\left[f_{i}\right]
$$

where $d(s)$ represents a monic characteristic polynomial with $n$ degrees; $n_{i}(s)$, for $i=1, \ldots, p$, denote the numerator of the transfer function with appropriate degrees for $f_{i}$ and $y$. Let $r(s)$ as a monic Hurwitz polynomial, defined as

$$
r(s) \equiv(s+r) \lambda(s)
$$

where $r>0 ; \lambda(s) \equiv \lambda_{c}^{\mathrm{T}} \alpha_{n-1}(s)$ with $\alpha_{n-1}(s) \equiv$ $\operatorname{col}\left[s^{n-1}, s^{n-2}, \ldots, s, 1\right]$ and $\lambda_{c} \equiv \operatorname{col}\left[1, \lambda_{n-2}, \ldots, \lambda_{1}, \lambda_{0}\right]$, $\lambda_{j}>0$, for $j \leq n-2$. By adding and substracting $\sum_{i=1}^{p}\left(n_{i}(s) / r(s)\right)\left[f_{i}\right]$ to the right-hand side of (3), the following is obtained:

$$
\begin{aligned}
y= & \frac{1}{s+r} \\
& \left\{\sum_{i=1}^{p} \frac{n_{i}(s)}{\lambda(s)}\left[f_{i}\right]+\frac{r(s)-d(s)}{\lambda(s)}\left(\sum_{i=1}^{p} \frac{n_{i}(s)}{d(s)}\left[f_{i}\right]\right)\right\} .
\end{aligned}
$$

Let $n_{p+1}(s) \equiv r(s)-d(s)$ and $f_{p+1}(t) \equiv y(t)$, then the transfer function (3) will have a Hurwitz denominator represented as

$$
y=\frac{1}{s+r} \sum_{i=1}^{p+1} \frac{\phi_{i}^{\mathrm{T}} \alpha_{n-1}(s)}{\lambda(s)}\left[f_{i}\right]
$$

where $\phi_{i} \in R^{n}$ is the coefficient vector of the polynomial $n_{i}(s)$, for $i=1, \ldots, p+1$. To find a state-space representation of (5) as (2), let us denote

$$
\begin{aligned}
\Lambda & =\left[\begin{array}{c:c}
-\lambda_{n-2}-\lambda_{n-3} \cdots & -\lambda_{0} \\
\hdashline I_{n-2} & 0
\end{array}\right] \in R^{(n-1) \times(n-1)} \\
B_{\lambda} & =\left[\begin{array}{c}
1 \\
0 \\
\vdots \\
0
\end{array}\right] \in R^{n-1} .
\end{aligned}
$$


We can see that $(s I-\Lambda)^{-1} B_{\lambda}=\alpha_{n-2}(s) / \lambda(s)$ and

$$
\frac{\phi_{i}^{\mathrm{T}} \alpha_{n-1}(s)}{\lambda(s)}=\vartheta_{i}+\theta_{i}^{\mathrm{T}}(s I-\Lambda)^{-1} B_{\lambda}
$$

where $\vartheta_{i}$ is the first element of $\phi_{i}$ and $\theta_{i}=\phi_{i}-\phi_{i, 1} \lambda$. Now based on transfer function (5), the system (1) is equivalently written as the state equation in (2) with $\vartheta=\operatorname{col}\left[\vartheta_{1}, \vartheta_{2}, \ldots, \vartheta_{p+1}\right], \theta=\operatorname{col}\left[\theta_{1}, \theta_{2}, \ldots, \theta_{p+1}\right]$. The constant matrix may be determined by differentiating the output equations of (1) and (2). Notice that the matrix $F$ is dependent only on system parameters.

Remark 1: For chaotic systems, the trajectory evolves either in a strange attractor, or on a periodic orbit/equilibrium point. Therefore, the states in the drive system are considered to be bounded chaotic signals. This implies that its equivalent realization $z$ is bounded also.

Since $(A, C)$ is observable, system (1) can be rewritten as follows:

$$
\dot{x}=(A-L C) x+\sum_{i=1}^{p+1} B_{i} f_{i}
$$

where $(A-L C)$ is Hurwitz; and $B_{p+1}=L, f_{p+1}=y$. Hence, we have an alternative representation for the dynamical system (1). From (3), it follows that

$$
y=\sum_{i=1}^{p+1} \frac{\phi_{i}^{\mathrm{T}} \alpha_{n-1}(s)}{d(s)}\left[f_{i}\right]
$$

which is thus implemented as the following state equation:

$$
\begin{aligned}
\dot{\omega}_{i}(t) & =\Lambda_{0} \omega_{i}+B_{0} f_{i}(t), \quad i=1, \ldots, p+1 \\
y(t) & =\phi^{T} \omega(t)
\end{aligned}
$$

where $\Lambda_{0} \in R^{n \times n}, B_{0} \in R^{n}$ which have the same structure as $\Lambda$ and $B_{\lambda}$, respectively; and satisfy $\left|s I-\Lambda_{0}\right|=|s I-A+L C|$, $\phi=\operatorname{col}\left[\phi_{1}, \ldots, \phi_{p+1}\right]$, and $\omega=\operatorname{col}\left[\omega_{1}, \ldots, \omega_{p+1}\right]$.

Proposition 2: The system $\sum_{1}$ is input-output equivalent to a $(p+1) n$ dimensional system (6). Furthermore, $x=F \omega$ for a constant matrix $F \in R^{n \times(p+1) n}$.

\section{B. Synchronizer Design With Exactly Known Dynamics}

According to Proposition 1 and 2, system (1) is input-output equivalent to (2) and (6) in different state-space representations. Thus, we have the following result of synchronization.

Proposition 3: Consider the drive system $\sum_{1}$ in (1) provided with a scalar driving signal $y$, there exists a response system

$$
\begin{aligned}
\sum_{2}: \quad \dot{\hat{z}}_{1}(t) & =-r \hat{z}_{1}(t)+\vartheta^{\mathrm{T}} f(y, t)+\theta^{\mathrm{T}} \hat{\omega}(t) \\
\dot{\hat{\omega}}_{i}(t) & =\Lambda \hat{\omega}_{i}(t)+B_{\lambda} f_{i}(y, t) \\
i=1, \ldots, p+1 ; \quad \hat{y} & =\hat{z}_{1}
\end{aligned}
$$

with a transformation matrix defined in Proposition 1, such that $\hat{x} \equiv F \hat{z}$, where $\hat{z}=\operatorname{col}\left[\hat{z}_{1}, \hat{\omega}\right]$ is exponentially synchronized to $x(t)$, i.e., $(\hat{x}-x)$ converges to zero exponentially.

The proof is a slight extension for the single input case in [20], [21]. In addition, it can be followed by the proof of Proposition 5 and thus is omitted here.

Similarly, according to (6), we have the following result.
Proposition 4: Consider the drive system $\sum_{1}$ in (1) with Hurwitz $A$, there exists a response system

$$
\begin{aligned}
\sum_{3}: \quad \dot{\hat{\omega}}_{i}(t) & =\Lambda_{0} \hat{\omega}_{i}(t)+B_{0} f_{i}(t), \quad i=1, \ldots, p+1 \\
\hat{y}(t) & =\phi^{T} \hat{\omega}(t)
\end{aligned}
$$

with a transformation matrix $F$ defined in Proposition 2 such that $\hat{x}=F \hat{\omega}$ will exponentially approach to $x$.

The proof follows from the fact that $\dot{\tilde{\omega}}_{i}(t)=\Lambda_{0} \tilde{\omega}_{i}$ and, thus, $\tilde{\omega}_{i}$ exponentially converges to zero.

Remark 2: To reduce the dimensions of the response system, $f_{i}$ may denote a combination of nonlinear terms and output $y$. We will emphasize this point in the examples in Section III.

Remark 3: In [21], a linear generalized synchronization is defined by a synchronizing manifold $\bar{H}=\{(x, \hat{z}) \mid \hat{z}=\bar{F} x\}$, with a constant matrix $\bar{F} \in R^{m \times n}$, and $m \geq n$. Since the independent variables are less than the constraints, it is shown that the generalized synchronization of chaos is achieved if and only if $m=n$ via linear transformations. For our design approach, the transformation matrix $F$ is existent based only on assuming the linear part of the drive system $\sum_{1}$ is controllable and observable. Therefore, the state of drive system $x$ can be reconstructed by $\hat{x}=F \hat{z}$ using a scalar signal $y$.

\section{Synchronization in Adaptive Case}

Using $y(t)$ as the scalar driving signal for synchronization, a suitable response system with exactly known parameters was developed in the above section. If there is uncertainty in system parameters, the designed response system $\sum_{2}$ and $\sum_{3}$ may lead to degraded performance and even cause instability. Here assuming the parameters of the chaotic systems are fully or partially unknown, an adaptive mechanism is utilized in order for the response system to synchronize the states of the drive system.

Since the drive-system (1) can be equivalently written as (2) [or (6)], the system output are rearranged in a linear combination of the injection inputs $f_{i}$ and their filtered signals. According whether the combinational parameters are known or not, we have the following expression:

$$
\vartheta^{T} f(y, t)+\theta^{T} \omega(t)=\varphi^{\mathrm{T}} \bar{\omega}(f, \omega)+\psi(f, \omega)
$$

where $\varphi$ is a $m$-dimensional vector containing the unknown or uncertain parameters; $\bar{\omega}$ is an $m \times 1$ vector which is linear in $f$ or $\omega$; and the sum of the remaining term are $\psi(f, \omega)$, which is also linear in $f$ or $\omega$. The properties for $\bar{\omega}$ and $\psi$ are

$$
\begin{aligned}
& \bar{\omega}(f, \hat{\omega})-\bar{\omega}(f, \omega)=\bar{\omega}(0, \tilde{\omega}) \\
& \psi(f, \hat{\omega})-\psi(f, \omega)=\psi(0, \tilde{\omega})
\end{aligned}
$$

where $\tilde{\omega}=\hat{\omega}-\omega$. The adaptive synchronizer proposed here is constructed by substituting an estimated parametric vector $\hat{\varphi}$ for the unknown actual parametric vector $\varphi$ in (9). Then, the adaptive synchronizer is designed as

$$
\begin{aligned}
\sum_{4}: & \dot{\hat{z}}_{1}(t)=-r \hat{z}_{1}(t)+\hat{\varphi}^{\mathrm{T}} \bar{\omega}(f, \hat{\omega})+\psi(f, \hat{\omega}) \\
\dot{\hat{\omega}}_{i}(t) & =\Lambda \hat{\omega}_{i}(t)+B_{\lambda} f_{i}(y, t), \quad i=1, \ldots, p+1 \\
\hat{y}(t) & =\hat{z}_{1}(t) .
\end{aligned}
$$


The error dynamics are

$$
\begin{aligned}
\dot{e} & =-r e+\tilde{\varphi}^{\mathrm{T}} \bar{\omega}(f, \hat{\omega})+\varphi^{\mathrm{T}} \bar{\omega}(0, \tilde{\omega})+\psi(0, \tilde{\omega}) \\
\dot{\tilde{\omega}}_{i} & =\Lambda \tilde{\omega}_{i}, \quad i=1, \ldots, p+1 .
\end{aligned}
$$

where $e=\hat{y}-y$ and $\tilde{\varphi}=\hat{\varphi}-\varphi$ denotes the estimated parameter error.

Proposition 5: Given a dynamic system (1) provided with a scalar driving signal $y$, the response system (12) is tuned by the update law

$$
\dot{\hat{\varphi}}(t)=-e \Gamma \bar{\omega}(f, \hat{\omega})
$$

with adaptation gain $\Gamma=\Gamma^{\mathrm{T}}>0$. Then, error signals $e, \tilde{\omega}_{i}$ in (13), (14) will asymptotically converge to zero.

Proof: Consider a Lyapunov function candidate as

$$
V_{2}=\frac{1}{2}\left(e^{2}+\tilde{\varphi}^{\mathrm{T}} \Gamma^{-1} \tilde{\varphi}+\delta^{\prime} \sum_{i=1}^{p+1} \tilde{\omega}_{i}^{\mathrm{T}} P \tilde{\omega}_{i}\right)
$$

where $\delta^{\prime}>0$ is a scalar to be chosen, $\Gamma=\Gamma^{\mathrm{T}}>0$ is an adaptation gain, and $P=P^{\mathrm{T}}>0$ satisfies the Lyapunov equation $P \Lambda+\Lambda^{\mathrm{T}} P=-I$. The time derivative of (16) is

$$
\dot{V}_{2}=-r e^{2}+e \varphi^{\mathrm{T}} \bar{\omega}(0, \tilde{\omega})+e \psi(0, \tilde{\omega})-\frac{\delta^{\prime}}{2} \sum_{i=1}^{p+1} \tilde{\omega}_{i}^{\mathrm{T}} \tilde{\omega}_{i}
$$

where the update law for tuning estimated parameters $\hat{\varphi}$ in (15) is applied. From the properties of $\bar{\omega}(\cdot)$ and $\psi(\cdot)$, it follows that $\|\bar{\omega}(0, \tilde{\omega})\| \leq \rho_{1}|| \tilde{\omega} \|$ and $|\psi(0, \tilde{\omega})| \leq \rho_{2}\|\tilde{\omega}\|$ for some constants $\rho_{1}, \rho_{2}>0$. Hence, $\dot{V}_{2}$ yields

$$
\dot{V}_{2} \leq-\frac{1}{2}\left[\begin{array}{ll}
|e| & \| \tilde{\omega}||
\end{array}\right]\left[\begin{array}{cc}
2 r & -\rho \\
-\rho & \delta^{\prime}
\end{array}\right]\left[\begin{array}{c}
|e| \\
\| \tilde{\omega}||
\end{array}\right]
$$

where $\rho=\|\varphi\| \rho_{1}+\rho_{2}$. If $\delta^{\prime}$ is chosen to be greater than $\rho^{2} / 2 r$, then $\dot{V}_{2} \leq 0$, which implies that $V_{2}$ is upper bounded and the origin of error systems (13) and (14) are uniformly stable in the large. Accordingly, $V_{2}$ is upper bounded and $\int_{0}^{\infty} \dot{V}_{2}(\tau) d \tau<$ $\infty$. Therefore, $e, \tilde{\omega} \in L_{2} \cap L_{\infty}$ and also $\dot{e}, \tilde{\omega} \in L_{\infty}$. This means $\lim _{t \rightarrow \infty} e, \tilde{\omega}=0$, which is derived from Barbalat's lemma [22].

For the representation (8), we have the following result.

Proposition 6: Given a dynamic system (1) provided with a scalar signal $y$, the response system

$$
\begin{aligned}
\sum_{5}: \quad \dot{\hat{\omega}}_{i}(t) & =\Lambda_{0} \hat{\omega}_{i}(t)+B_{0} f_{i}(t) \\
\hat{y}(t) & =\phi^{T} \hat{\omega}(t) .
\end{aligned}
$$

is tuned by update law

$$
\dot{\tilde{\phi}}=-e \hat{\omega}
$$

Then, $\tilde{\omega}_{i}$ will asymptotically converge to zero.

\section{Persistent Excitation and Exponential Synchronization}

To achieve $\lim _{t \rightarrow \infty} \hat{\varphi}=\varphi$ in Propositions 5 (or $\lim _{t \rightarrow \infty} \hat{\phi}=\phi$ in Proposition 6), and $\hat{x}=F(\hat{\varphi}) \hat{z}$ (or
$\hat{x}=F(\hat{\phi}) \hat{\omega})$ is synchronized to $x$ is related to the sufficient richness condition, or persistent excitation on $\bar{\omega}(f, \hat{\omega})$ [or $\hat{\omega}$ ]. It can be shown that $\tilde{\varphi}$ converges to zero exponentially if $\bar{\omega}$ is persistent exciting [19], i.e., there are positive constants $\varepsilon$ and $k$ such that

$$
\int_{t}^{t+\varepsilon} \bar{\omega}(\tau) \bar{\omega}^{\mathrm{T}}(\tau) d \tau \geq k I \quad \forall t \geq 0
$$

Compared to the implicit condition (18) on $\bar{\omega}$, we can give explicit conditions on $f_{i}$ to guarantee the exponential synchronization. From the definition of $\bar{\omega}$, we have

$$
\begin{aligned}
\bar{\omega} & =H\left(f_{1}, \hat{\omega}_{1}, \ldots, f_{p+1}, \hat{\omega}_{p+1}\right) \\
& =T w_{0}
\end{aligned}
$$

for an onto linear map $H$. In the expression, $T$ denotes the matrix representation of $H$, which is full row-rank. Notice that $f_{i}$ will be absent in $w_{0}$ if $n_{i}(s) / \lambda(s)$ is strictly proper. Let $q_{i}$ denote the dimensions of $\left[f_{i}, \omega_{i}\right]$ or $\omega_{i}$ if $n_{i}(s) / \lambda(s)$ is strictly proper. Then, $q=q_{1}+\cdots+q_{p+1}$ is the dimension of $w_{0}$. According to the results in [19], [20], the PE condition on $\bar{\omega}$ is equivalent to the PE condition on $w_{0}$, which can be verified via

C.1) $w_{0}$ has at least $q$ spectral lines;

C.2) at these $q$ spectral lines, $\left\{\hat{W}_{0}\left(\nu_{1}\right), \ldots, \hat{W}_{0}\left(\nu_{q}\right)\right\}$ are linearly independent in $C^{q}$.

The signal $w_{0}(t): R^{+} \rightarrow R^{n}$ is said to have a spectral line at frequency $\nu$ of amplitude $\hat{W}_{0}(\nu)(\neq 0) \in C^{q}$ if and only if $(1 / T) \int_{s}^{s+T} w_{0}(t) s^{-j \nu t} d t$ converges to $\hat{W}_{0}(\nu)$ uniformly in $s$ as $T \rightarrow \infty$ [20]. Since $(s I-\Lambda)^{-1} B_{\lambda}$ is a stable filter, it follows that $w_{i}$ and $f_{i}$ have the same spectral lines [20]. Therefore, we have the following result.

Theorem 1: The trajectory of $\sum_{4}$, i.e., $\hat{x}=F(\hat{\varphi}) \hat{z}$, is exponentially synchronized to $\sum_{1}$ if the following conditions are satisfied:

1) $\left\{f_{1}, \ldots, f_{p+1}\right\}$ are linearly independent;

2) For each $f_{i}$ there exist $q_{i}$ spectral lines, such that the total $q_{1}+\cdots+q_{p+1}$ spectral lines are mutually different.

Since $y$ is a chaotic signal, the broad spectrum implies that C.1) is satisfied. The condition (C.2) is satisfied if the conditions 1) and 2) in Thm. 1 are satisfied. Similar results can be obtained for the representation $\sum_{5}$. In applying this scheme, the system matrix $A$ must be exactly known (unknown parameters exist in $B_{i}$ ). This condition generally leads to requiring a larger dimension of $\hat{z}$ to implement the response system.

By the stability analysis of the adaptive response system $\sum_{4}$, the convergence rate of the error signals $e, \tilde{\omega}$ is determined by the chosen parameters $r$ and $\lambda_{j}$ in (13) and (14), respectively. Note that when $r$ is given a larger value and $\Lambda$ are given with larger eigenvalues, the error signals $e$ and $\tilde{\omega}$ will converge faster to zero. This, on the other hand, will slow down the updating rate of estimated parameter $\hat{\varphi}$. From the viewpoint of the broad spectrum, noise-like and high sensitivity to parametric variation characteristic of chaotic signals, the persistently exciting condition would be easily satisfied if Condition 1) in Theorem 1 is satisfied. Then the adaptation gain $\Gamma$ may be properly chosen to simultaneously synchronize all states of the drive system by $F(\hat{\varphi}) \hat{z}$. Accordingly, a trade-off condition exists between the convergence rate of errors $e, \tilde{\omega}$ and parametric errors $\tilde{\varphi}$. 


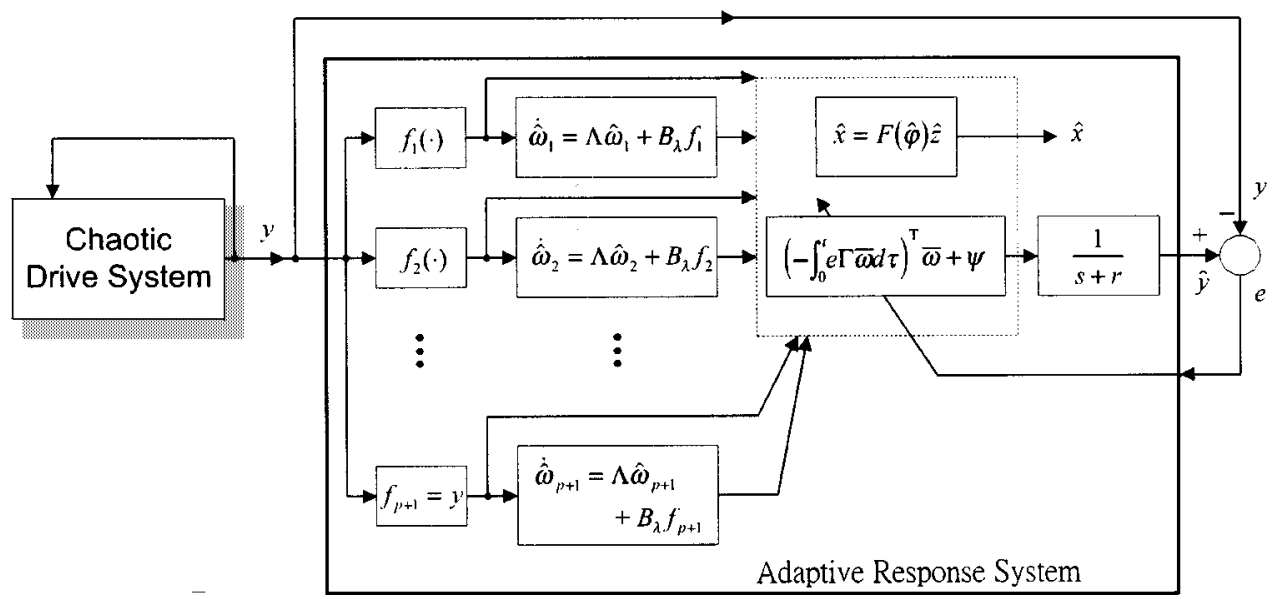

Fig. 1. Structure of adaptive synchronization with drive and response system using a scalar coupling channel.

The proposed design methodology for $\sum_{4}$ is summarized in the following adaptive synchronization algorithm.

Step 1) Determine the driving signal and the terms $f_{i}$. Then represent the chaotic system in the output injection form (1).

Step 2) Check, if the system matrix pair $(A, C)$ is observable and $\left(A, B_{i}\right)$ is controllable. When this is the case, the individual proper transfer functions from $f_{i}$ to $y$ can be found. The input-output description of the overall system is the sum of the individual transfer functions.

Step 3) Give $r(s)$ and define $f_{p+1}=y$ and $n_{p+1}(s)=$ $r(s)-d(s)$, then the transfer function is represented with a Hurwitz denominator. Express the filtered nonlinearity as $\vartheta^{\mathrm{T}} f(y, t)+\theta^{\mathrm{T}} \omega(t)$. The chaotic system is equivalent to (2). Meanwhile, the transformation matrix $F$ is calculated.

Step 4) Reparameterize $\vartheta^{\mathrm{T}} f(y, t)+\theta^{\mathrm{T}} \omega(t)$ as linear in terms of the unknown parametric set, i.e., $\varphi^{T} \bar{\omega}+\psi(f, \omega)$. Then the response system $\sum_{4}$ is designed with adaptation law (15). The states of the drive system are constructed by $\hat{x}=F(\hat{\varphi}) \hat{z}$.

This leads to the results in Theorem 1 . The overall structure to implement the response system is illustrated in Fig. 1.

\section{ApPliCATIONS ON TyPiCAL ChaOtic Systems}

To verify the validity of the adaptive synchronization methodology, two examples are considered with numerical simulations carried out.

Duffing-Holmes System: Consider the Duffing-Holmes system given

$$
\begin{aligned}
\dot{x}_{1} & =x_{2} \\
\dot{x}_{2} & =-p_{1} x_{1}-p_{2} x_{2}-p_{3} x_{1}^{3}+q \sin \left(w t+\theta_{0}\right) \\
y & =x_{1} .
\end{aligned}
$$

The parameters $p_{1}, p_{2}, p_{3}>0$ and the amplitude of the oscillation force $q$ are chosen so that the system exhibits chaotic behavior [23], [24]. Since the nonlinear terms $x_{1}^{3}$ and the forced sinusoid signal $\sin \left(w t+\theta_{0}\right)$ are dependent on output and time, respectively, the Duffing equation can be represented in the form (1). If all parameters are exactly known, we can rewrite (19) as

$$
\begin{aligned}
& \dot{x}=A x+B f(y, t) \\
& y=C x
\end{aligned}
$$

where

$$
\begin{aligned}
& A=\left[\begin{array}{cc}
0 & 1 \\
-p_{1} & -p_{2}
\end{array}\right] \quad B=\left[\begin{array}{l}
0 \\
1
\end{array}\right] \quad C=\left[\begin{array}{ll}
1 & 0
\end{array}\right] \\
& x=\left[\begin{array}{l}
x_{1} \\
x_{2}
\end{array}\right]
\end{aligned}
$$

and $f(y, t)=-p_{3} x_{1}^{3}+q \sin \left(\left(\omega t+\theta_{0}\right)\right.$. Thus $n=2, p=1$, and $A$ is Hurwitz. According to Proposition 4, we can design a second-order response system to achieve synchronization. Alternatively, a higher order (i.e., third order) response system can achieve synchronization if Proposition 3 is applied. To demonstrate adaptive synchronization, we now assume parameters $p_{1}$, $p_{2}, p_{3}$ and $q$ are unknown to the response system. In addition, the synchronizing time $t$ may be unknown initially such that the oscillation force may not be obtained. Hence we assume there is an unknown phase $\theta_{0}$ in the forced sinusoid signal. Note that the forced oscillation frequency is provided.

Let $f_{1}\left(x_{1}\right) \equiv-x_{1}^{3}, f_{2}(t) \equiv \sin w t$, and $f_{3}(t) \equiv \cos w t$. Then, the output injection form is obtained

$$
\begin{aligned}
\dot{x}= & {\left[\begin{array}{cc}
0 & 1 \\
-p_{1} & -p_{2}
\end{array}\right] x+\left[\begin{array}{c}
0 \\
p_{3}
\end{array}\right] f_{1}(y)+\left[\begin{array}{c}
0 \\
p_{4}
\end{array}\right] f_{2}(t) } \\
& +\left[\begin{array}{c}
0 \\
p_{5}
\end{array}\right] f_{3}(t) \\
y= & {\left[\begin{array}{ll}
1 & 0
\end{array}\right] x }
\end{aligned}
$$

where $p_{4} \equiv q \cos \left(\theta_{0}\right), p_{5} \equiv q \sin \left(\theta_{0}\right)$. In light of this, the input-output transfer function is therefore represented as

$$
\begin{aligned}
y(s)=\frac{p_{3}}{s^{2}+p_{2} s+p_{1}} f_{1}(s)+ & \frac{p_{4}}{s^{2}+p_{2} s+p_{1}} f_{2}(s) \\
& +\frac{p_{5}}{s^{2}+p_{2} s+p_{1}} f_{3}(s) .
\end{aligned}
$$


As stated in Section II-A, it is given $r(s)=(s+r)(s+\lambda)$ with $r, \lambda>0$, and $f_{4}=y, n_{4}(s)=r(s)-\left(s^{2}+p_{2} s+p_{1}\right)$. Therefore, (20) is rewritten as

$$
y(s)=\frac{1}{s+r} \sum_{i=1}^{4} \frac{\phi_{i}^{\mathrm{T}} \alpha_{1}(s)}{s+\lambda} f_{i}(s)
$$

where $\phi_{1}=\operatorname{col}\left[0, p_{3}\right], \phi_{2}=\operatorname{col}\left[0, p_{4}\right], \phi_{3}=\operatorname{col}\left[0, p_{5}\right]$, and $\phi_{4}=\operatorname{col}\left[\lambda+r-p_{2}, \lambda r-p_{1}\right]$. The input-output equivalent system is

$$
\begin{aligned}
\dot{z}_{1} & =-r z_{1}+\sum_{i=1}^{4} \vartheta y+\theta^{\mathrm{T}} \omega \\
\dot{\omega}_{i} & =-\lambda \omega_{i}+f_{i}, \quad i=1,2,3,4, \\
y & =z_{1}
\end{aligned}
$$

where $\vartheta=\lambda+r-p_{2}, \theta=\operatorname{col}\left[p_{3}, p_{4}, p_{5}, \lambda p_{2}-p_{1}-\lambda^{2}\right]$, and $\omega \equiv \operatorname{col}\left[\omega_{1}, \omega_{2}, \omega_{3}, \omega_{4}\right]$. Let $z \equiv \operatorname{col}\left[z_{1}, \omega\right]$. Therefore, $x=F z$ with

$$
F=\left[\begin{array}{cc}
1 & 0_{1 \times 4} \\
\lambda-p_{2} & \theta^{\mathrm{T}}
\end{array}\right] .
$$

The response system with known parameters can be obtained from (8). Furthermore, $\vartheta y+\theta^{\mathrm{T}} \omega$ can be reparameterized in terms of an unknown parametric set as $\varphi^{T} \bar{\omega}+\psi$ where $\varphi=$ $\operatorname{col}\left[p_{1}, p_{2}, \ldots, p_{5}\right] ; \bar{\omega}=\operatorname{col}\left[-\omega_{4}, \lambda \omega_{4}-y, \omega_{1}, \omega_{2}, \omega_{3}\right]$ and $\psi=(\lambda+r) y-\lambda^{2} \omega_{4}$. According to adaptive synchronization for the general case addressed in (12), the response system is

$$
\begin{aligned}
\dot{\hat{z}}_{1} & =-r \hat{z}_{1}+\hat{\varphi}^{\mathrm{T}} \bar{\omega}(f(y), \hat{\omega})+\psi(f(y), \hat{\omega}) \\
\dot{\hat{\omega}}_{i} & =-\lambda \hat{\omega}_{i}+f_{i}(y, t), \quad i=1,2, \ldots, 4 \\
\hat{y} & =\hat{z}_{1}
\end{aligned}
$$

where the update law for the estimated parameter is $\dot{\hat{\varphi}}=-e \Gamma \bar{\omega}(f(y), \hat{\omega})$. Also, the state $x_{2}$ can be reconstructed by $\hat{x}=F(\hat{\varphi}) \hat{z}$.

Consider system (19) synchronized by the adaptive response system (21). Notice that in the adaptive response system, $f_{1} \sim f_{4}$ are linearly independent whereas $f_{1}, f_{4}$ have broad spectrum and $f_{2}, f_{3}$ have two spectrum lines, respectively. The PE conditions in Thm. 1 is satisfied and hence exponential synchronization is achievable. For simulations, the parametric values $p_{1}=$ $-1.1, p_{2}=0.4, p_{3}=1$, and $q=1.8$. The oscillation force has the given frequency $w=1.8 \mathrm{rad} / \mathrm{s}$ and a phase $\theta_{0}=\pi / 3$. The initial states $x_{1}(0)=0.2$, and $x_{2}(0)=0.1$. For the response system (21), the parameters are chosen as $r=\lambda=3$. The initial values of the states and estimated parameters are both set as zero, i.e., $\hat{z}(0)=0$, and $\hat{\varphi}(0)=0$. The adaptation gain is chosen as $\Gamma=\operatorname{diag}\{20,45,13,55,35\}$. Fig. 2(a) and (b) illustrates the errors $\hat{x}_{1}-x_{1}$ and $\hat{x}_{2}-x_{2}$, respectively. The errors converge to zero as estimated parameters converge to the true value. Fig. 3 shows the response of the estimated parameters $\hat{p}_{1} \sim \hat{p}_{5}$.
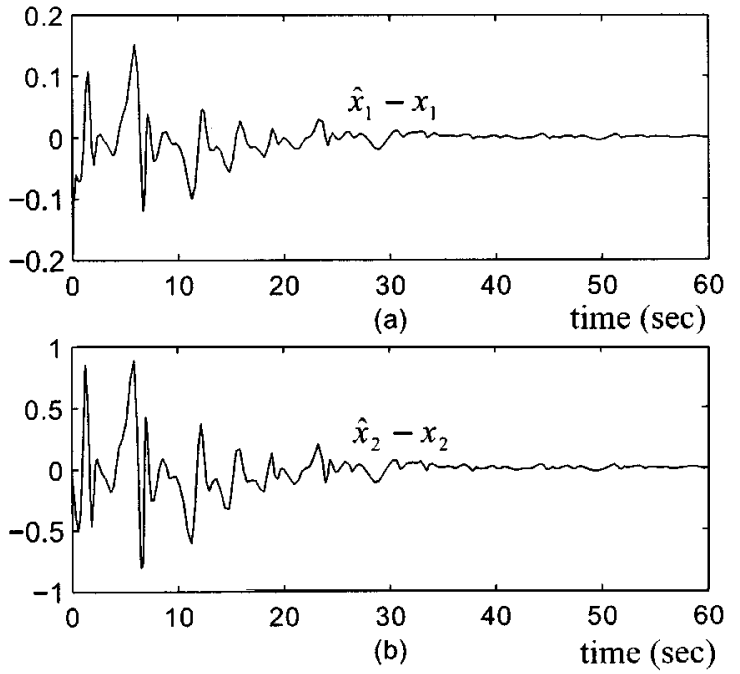

Fig. 2. (a) Synchronization error $\hat{x}_{1}-x_{1}$ of Duffing system; (b) synchronization error $\hat{x}_{2}-x_{2}$ of Duffing system.

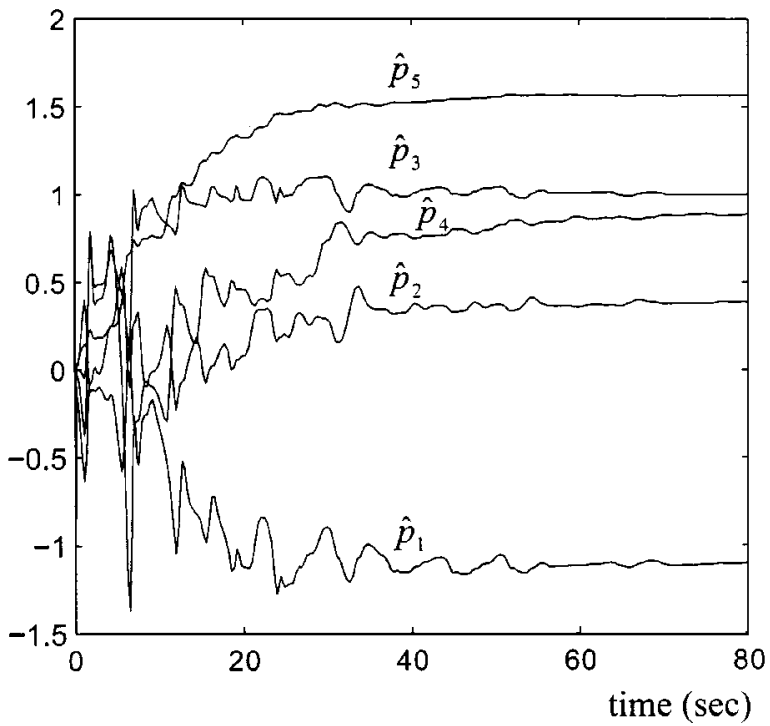

Fig. 3. Time response of estimated parameters $\hat{p}_{1} \sim \hat{p}_{5}$ in adaptive response system for Duffing system.

Chua's Circuit: Consider the Chua's circuit described by the following equations:

$$
\begin{aligned}
\dot{x}_{1} & =a_{1}\left(-x_{1}+x_{2}-\bar{f}\left(x_{1}\right)\right) \\
\dot{x}_{2} & =x_{1}-x_{2}+x_{3} \\
\dot{x}_{3} & =-a_{2} x_{2} \\
y & =x_{1}
\end{aligned}
$$

where $\bar{f}\left(x_{1}\right)$ is the nonlinear term that represents the voltage-current characteristics of the nonlinear resistor in the circuit. With $g_{a}$ and $g_{b}$ as the different slopes associated with the break-off points in the voltage-current plot for the nonlinear resistor, $\bar{f}\left(x_{1}\right)$ is given by

$$
\bar{f}\left(x_{1}\right)=g_{b} x_{1}+\frac{1}{2}\left(g_{a}-g_{b}\right)\left(\left|x_{1}+1\right|-\left|x_{1}-1\right|\right)
$$


where parameters $a_{1}, a_{2}$ and nonlinearity $\bar{f}\left(x_{1}\right)$ of the circuit are known to exhibit many forms of chaotic behavior in [15], [25].

If all the parameters are exactly known, we can rewrite (22) as

$$
\begin{aligned}
& \dot{x}=A x+B f(y) \\
& y=C x
\end{aligned}
$$

with

$$
\begin{aligned}
& A=\left[\begin{array}{ccc}
-a_{1} & a_{1} & 0 \\
1 & -1 & 1 \\
0 & -a_{2} & 0
\end{array}\right] \quad B=\left[\begin{array}{c}
a_{1} \\
0 \\
0
\end{array}\right] \\
& C=\left[\begin{array}{lll}
1 & 0 & 0
\end{array}\right] \quad x=\left[\begin{array}{lll}
x_{1} & x_{2} & x_{3}
\end{array}\right]^{T} .
\end{aligned}
$$

and $f(y)=\bar{f}\left(x_{1}\right)$. For typical values of $a_{1}$ and $a_{2}, A$ is Hurwitz. Hence we can design a third-order response system to achieve synchronization according to Proposition 4. For unknown parameters, the scheme in Proposition 5 will be applied. First, we rewrite Chua's circuit as

$$
\begin{aligned}
& \dot{x}=\left[\begin{array}{ccc}
-a_{3} & a_{1} & 0 \\
1 & -1 & 1 \\
0 & -a_{2} & 0
\end{array}\right] x+\left[\begin{array}{c}
a_{4} \\
0 \\
0
\end{array}\right] f_{1}(y) \\
& y=\left[\begin{array}{lll}
1 & 0 & 0
\end{array}\right] x
\end{aligned}
$$

where $x=\operatorname{col}\left[x_{1}, x_{2}, x_{3}\right] ; a_{3}=a_{1}\left(1+g_{b}\right) ; a_{4}=$ $-\left(a_{1} / 2\right)\left(g_{a}-g_{b}\right)$; and $f_{1}\left(x_{1}\right)=\left|x_{1}+1\right|-\left|x_{1}-1\right|$. The input-output transfer function of (23) is therefore

$$
y(s)=\frac{a_{4}\left(s^{2}+s+a_{2}\right)}{s^{3}+\left(a_{3}+1\right) s^{2}+\left(a_{3}-a_{1}+a_{2}\right) s+a_{2} a_{3}} f_{1}(s) .
$$

By $r(s)=(s+r)(s+\lambda)^{2}$ and defining $f_{2}=y$, an input-output equivalent representation is

$$
\begin{aligned}
\dot{z}_{1} & =-r z_{1}+\vartheta^{\mathrm{T}} f(y)+\theta^{\mathrm{T}} \omega \\
\dot{\omega}_{i} & =\left[\begin{array}{cc}
-2 \lambda & -\lambda^{2} \\
1 & 0
\end{array}\right] \omega_{i}+\left[\begin{array}{l}
1 \\
0
\end{array}\right] f_{i}(y), \quad i=1,2 \\
& \equiv \Lambda \omega_{i}+B_{\lambda} f_{i}(y) \\
y & =z_{1},
\end{aligned}
$$

where $\vartheta=\operatorname{col}\left[a_{4}, r+2 \lambda-a_{3}-1\right], f(y)=\operatorname{col}\left[f_{1}, f_{2}\right], \omega=$ $\operatorname{col}\left[\omega_{1}, \omega_{2}\right]$, and $\theta=\operatorname{col}\left[\theta_{1}, \theta_{2}\right]$ with

$$
\begin{gathered}
\theta_{1} \equiv \operatorname{col}\left[a_{4}(1-2 \lambda), \quad a_{4}\left(a_{2}-\lambda^{2}\right)\right] \\
\theta_{2} \equiv \operatorname{col}\left[2 \lambda\left(a_{3}+1\right)-3 \lambda^{2}+a_{1}-a_{2}-a_{3},\right. \\
\left.\lambda^{2}\left(a_{3}+1\right)-2 \lambda^{3}-a_{2} a_{3}\right] .
\end{gathered}
$$

According to $z=\operatorname{col}\left[z_{1}, \omega\right]$ and the original state equation (23), the transformation matrix can be found in the form

$$
F=\frac{1}{a_{1}}\left[\begin{array}{cc}
a_{1} & 0_{1 \times 4} \\
2 \lambda-1 & \theta^{\mathrm{T}} \\
\lambda^{2}-a_{2} & \lambda^{2} \theta^{\mathrm{T}}+\theta^{\mathrm{T}} \bar{\Lambda}
\end{array}\right]_{3 \times \tilde{5}}
$$

where $\bar{\Lambda}=\operatorname{diag}\{\Lambda, \Lambda\}$. Hence, the adaptive response system is expressed as (12)

$$
\begin{aligned}
& \dot{\hat{z}}_{1}=-r \hat{z}_{1}+\hat{\varphi}^{\mathrm{T}} \bar{\omega}(f(y), \hat{\omega})+\psi(f(y), \hat{\omega}) \\
& \dot{\hat{\omega}}_{i}=\left[\begin{array}{cc}
-2 \lambda & -\lambda^{2} \\
1 & 0
\end{array}\right] \hat{\omega}_{i}+\left[\begin{array}{l}
1 \\
0
\end{array}\right] f_{i}, \quad i=1,2 \\
& \hat{y}=\hat{z}_{1}
\end{aligned}
$$

where $\psi=(r+2 \lambda-1) y-\left(3 \lambda^{2}-2 \lambda\right) \hat{\omega}_{21}-\left(2 \lambda^{3}-\lambda^{2}\right) \hat{\omega}_{22}$; and $\bar{\omega}=\operatorname{col}\left[\bar{\omega}_{1}, \bar{\omega}_{2}\right]$ with

$$
\begin{aligned}
& \bar{\omega}_{1}=\operatorname{col}\left[f_{1}(y)+(1-2 \lambda) \hat{\omega}_{11}-\lambda^{2} \hat{\omega}_{12}, \hat{\omega}_{12}\right] \\
& \bar{\omega}_{2}=\operatorname{col}\left[(2 \lambda-1) \hat{\omega}_{21}+\lambda^{2} \hat{\omega}_{22}-y, \quad \hat{\omega}_{21}, \quad-\hat{\omega}_{22}\right] .
\end{aligned}
$$

The vector $\hat{\varphi}$ is tuned by (15) to estimate the actual parametric set as

$$
\varphi=\operatorname{col}\left[a_{4}, \quad a_{2} a_{4}, \quad a_{3}, \quad\left(a_{1}-a_{2}\right), \quad a_{2} a_{3},\right] .
$$

To this step, we observe that both $\hat{\omega}_{12}$ and $\hat{\omega}_{22}$, which are driven by $f_{1}(y)$ and $y$, respectively, appear in $\bar{\omega}$. Since $f_{1}(y)$ and $y$ are linear dependent when $y$ evolves in the region $(-1,1)$, the degree of PE will be weakened and hence degrade the convergent rate of synchronization errors. To obtain a lower order response system and rapid synchronization, we assume $a_{2}$ is known. Then, the adaptive response system has the same expression as (24) with the following definitions:

$$
\begin{aligned}
\psi & =(r+2 \lambda-1) y-\left(3 \lambda^{2}-2 \lambda+a_{2}\right) \hat{\omega}_{12}-\left(2 \lambda^{3}-\lambda^{2}\right) \hat{\omega}_{22} \\
\bar{\omega}_{1} & =f_{1}(y)+(1-2 \lambda) \hat{\omega}_{11}+\left(a_{2}-\lambda^{2}\right) \hat{\omega}_{12} \\
\bar{\omega}_{2} & =\operatorname{col}\left[(2 \lambda-1) \hat{\omega}_{21}+\left(\lambda^{2}-a_{2}\right) \hat{\omega}_{22}-y, \hat{\omega}_{21}\right] \\
\varphi & =\operatorname{col}\left[a_{4}, \quad a_{3}, a_{1}\right] .
\end{aligned}
$$

This yields an eighth-order response system. In addition, applying estimated parameters $\hat{\varphi}$ to replace the actual parameters in matrix $F, F(\hat{\varphi}) \hat{z}$ is used to synchronize the state $x$. Thus, perfect synchronization is achieved once the PE condition is satisfied.

For Chua's circuit (22), we set the unknown parameters as $a_{1}=10, g_{a}=-1.27$, and $g_{b}=-0.68$, and the initial states are chosen as $x(0)=\operatorname{col}[-1,0,0]$. The known parameter $a_{2}$ is set as 14.87. For the response system, the parameters are set as $r=100, \lambda=5$ and the initial conditions are set as $\hat{z}(0)=0$. The initial values of the estimated parameters can be given arbitrarily. Here, we set them as zero except for the estimated $a_{1}=$ 15. The adaptation gain is chosen as $\Gamma=\operatorname{diag}\{40.5,3,39\}$. The synchronization error $\tilde{x}_{1}(t)$ are shown in Fig. 4 , which show that trajectories of the drive and response systems achieve synchronization. The estimated parameters of $\hat{a}_{1}, \hat{a}_{3}, \hat{a}_{4}$ shown in Fig. 5 converges close to true values, which are 10,3.2, and 2.95, respectively.

\section{Disturbance AND Robustness}

The robustness issue of the proposed scheme due to bounded disturbance is discussed in this section. In other words, we con- 


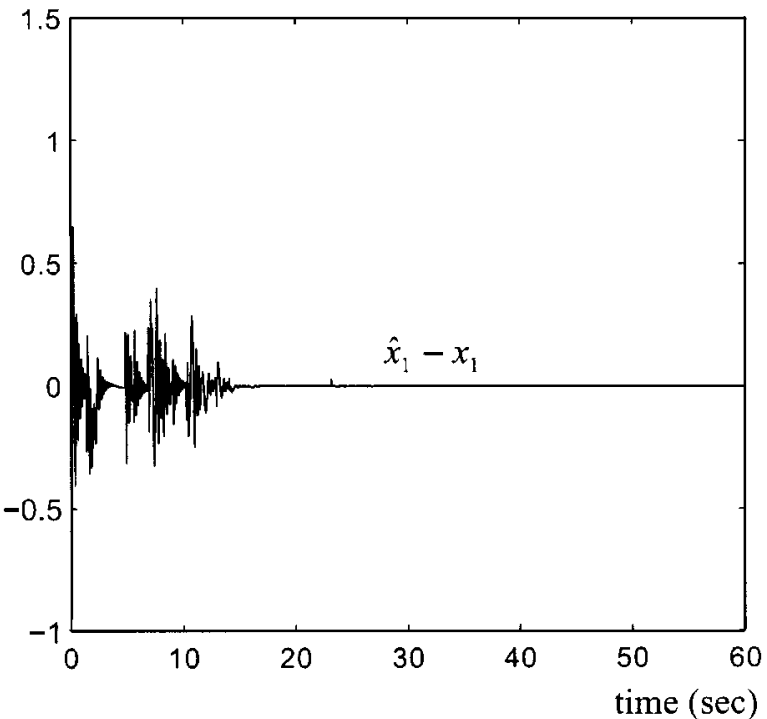

Fig. 4. Synchronization error $\hat{x}_{1}-x_{1}$ of Chua's circuit.

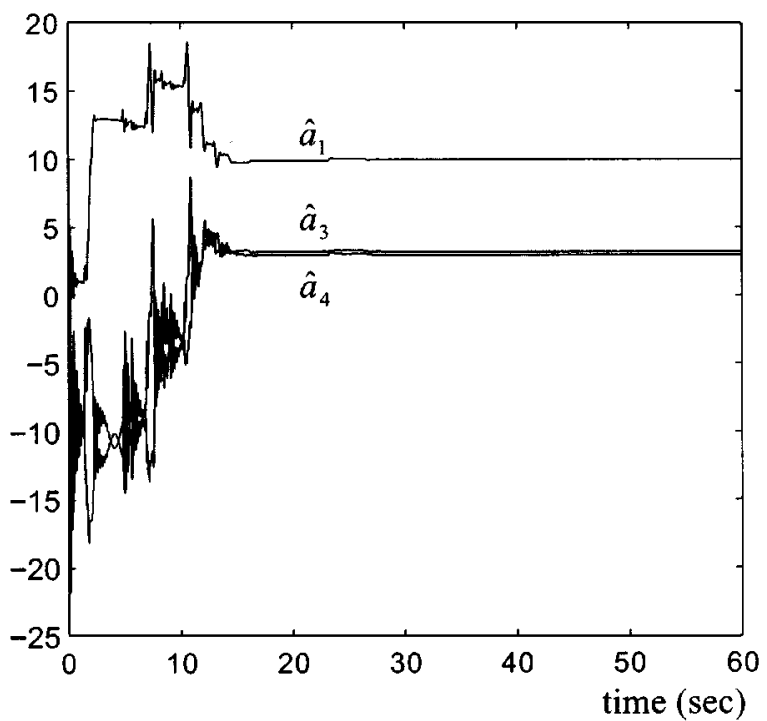

Fig. 5. Time response of estimated parameters $\hat{a}_{1}, \hat{a}_{3}, \hat{a}_{4}$ in adaptive response system for Chua's circuit.

sider the response system is under influence of external disturbances. The synchronization error and updating parameters would not converge to zero in this case. In the worst case scenario, these performance objectives may grow to be unbounded. The disturbance may arise from the following two cases.

Case i): - The adaptive response system given in (12) is driven by a scalar output signal $y$ from the drive system. The measurement of output $y(t)$ is easily corrupted with noise $n(t)$, i.e., the noisy output $\bar{y}(t)=y(t)+n(t)$. This noisy output will drive the adaptive response system (12). Therefore, we arrive at the following error dynamics:

$$
\begin{aligned}
\dot{e}(t) & =-r e+\tilde{\varphi} \bar{\omega}(f, \hat{\omega})+\varphi \bar{\omega}(0, \tilde{\omega})+\psi(0, \tilde{\omega})+e_{n} \\
\dot{\tilde{\omega}} & =\Lambda \tilde{\omega}_{i}+\omega_{n i} \quad \forall i=1, \ldots, p \\
\dot{\tilde{\varphi}} & =-e \Gamma \bar{\omega}(f, \hat{\omega})+\varphi_{n}
\end{aligned}
$$

where $e_{n}=\hat{\varphi} \bar{\omega}(n, 0)+\psi(n, 0), \omega_{n i}=B_{\lambda}\left(f_{i}(y+n)-f_{i}(y)\right)$, and $\varphi_{n}=n \Gamma \bar{\omega}$ are the additional terms due to the noise.
Case ii): - The adaptive synchronization scheme has a potential application on transmitting messages via unknown parameters (see comments addressed in Section V). In this case, the unknown parameters varies slowly with respect to time such that $\dot{\varphi}$ can be considered as a bounded disturbance. Then the error dynamics have the same form as (25) with $e_{n}=\omega_{n i}=0$ and $\varphi_{n}=\dot{\varphi}$.

As stated above, Case ii) can be regarded as a special case for Case i). Hence we take (25) as a general error dynamics for the adaptive synchronization subject to bounded disturbance. Let $\underline{\dot{e}}=[e, \tilde{\omega}, \tilde{\varphi}]$. The error dynamics (25) can be written as

$$
\underline{\dot{e}}=\underline{A} \cdot \underline{e}+d(t, \underline{e})
$$

with

$$
\underline{A}=\left[\begin{array}{ccc}
-r & \underline{A}_{12} & \bar{\omega} \\
0 & \Lambda & 0 \\
-\Gamma \bar{\omega} & 0 & 0
\end{array}\right] \quad d=\left[\begin{array}{c}
e_{n} \\
\omega_{n} \\
\varphi_{n}
\end{array}\right]
$$

where $\omega_{n}=\operatorname{col}\left[\omega_{n 1}, \ldots, \omega_{n p}\right]$ and $\underline{A}_{12}=(\varphi \bar{\omega}(0, \tilde{\omega})+$ $\psi(0, \tilde{\omega})) / \tilde{\omega}$. We notice that (26) is exactly same as the error dynamics considered in Thm. 1 if the disturbance $d$ equals zero. Suppose the PE condition holds, then the trajectories of $\underline{\dot{e}}=\underline{A e}$ converge to zero exponentially. From the converse Lyapunov theorem [22], there exists a function $V(t, \underline{e})$ that satisfies the inequalities

$$
\begin{aligned}
c_{1}\|\underline{e}\|^{2} & \leq V(t, \underline{e}) \leq c_{2}\|\underline{e}\|^{2} \\
\frac{\partial V}{\partial t}+\frac{\partial V}{\partial \underline{e}} \underline{A} \cdot \underline{e} & \leq-c_{3}\|\underline{e}\|^{2} \\
\left\|\frac{\partial V}{\partial \underline{e}}\right\| & \leq c_{4}\|\underline{e}\|
\end{aligned}
$$

for some positive constants $c_{1}, c_{2}, c_{3}$, and $c_{4}$. The disturbance $d$ satisfies the inequality

$$
\|d(t, \underline{e})\| \leq r_{n}(t)\|\underline{e}\|+\delta_{n}(t)
$$

where the magnitudes of $r_{n}$ and $\delta_{n}$ are proportional to $n(t)$. The derivative of $V(t, \underline{e})$ along the trajectories of (26) satisfies

$$
\begin{aligned}
\dot{V}(t, \underline{e}) & =\frac{\partial V}{\partial t}+\frac{\partial V}{\partial \underline{e}} \underline{A} \cdot \underline{e}+\frac{\partial V}{\partial \underline{e}} d(t, \underline{e}) \\
& \leq-\left[\frac{c_{3}}{c_{2}}-\frac{c_{4}}{c_{1}} r_{n}(t)\right] V+c_{4} \delta_{n}(t) \sqrt{\frac{V}{c_{1}}} .
\end{aligned}
$$

Let $W(t)=\sqrt{V}$ and therefore $\dot{W}(t)=\dot{V} / 2 \sqrt{V}$, we have

$$
\dot{W} \leq-\frac{1}{2}\left[\frac{c_{3}}{c_{2}}-\frac{c_{4}}{c_{1}} r_{n}(t)\right] W+\frac{c_{4}}{2 \sqrt{c_{1}}} \delta_{n}(t) .
$$

If $n(t)$ is small enough such that $\left(c_{4} / c_{1}\right) r_{n}(t)$ is dominated by $c_{3} / c_{2}$, then we obtain, after the transient time, $\|\underline{e}(t)\|$ bounded above by $\alpha \sup _{t \geq 0} \delta_{n}(t)$ for some constant $\alpha$.

Theorem 2: If the (PE) conditions in Theorem 1 are satisfied, the synchronization error remains bounded if the noise is small enough. Furthermore, the ultimate bound is proportional to the magnitude of noise. 

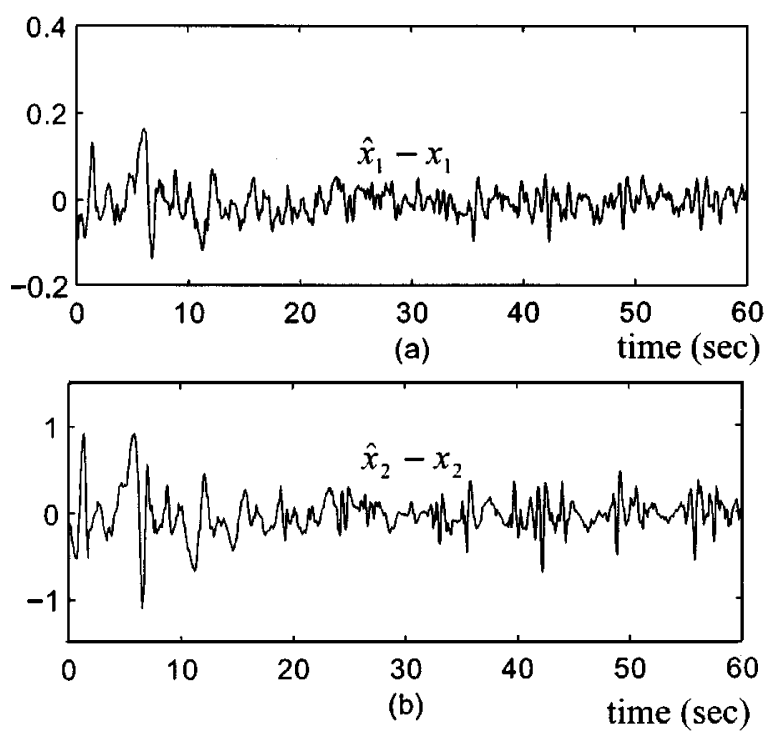

Fig. 6. (a) Synchronization error $\hat{x}_{1}-x_{1}$ of Duffing system with noisy coupling signal using robust adaptive law. (b) Synchronization error $\hat{x}_{2}-x_{2}$ of Duffing system with noisy coupling signal using robust adaptive law.

Note that for signals having a higher degree of PE, the faster the convergence for $\|\underline{e}(t)\|$. In this case, the tolerance of amount of disturbance is also larger. An alternative method to enlarge the tolerance of disturbance is to modify the update law for the unknown parameters in the following form:

$$
\dot{\hat{\varphi}}=-e_{0} \Gamma \bar{\omega}(f(\bar{y}), \hat{\omega})-\sigma \Gamma \hat{\varphi}
$$

where $\sigma>0$ and $e_{0}(t) \equiv \hat{y}(t)-\bar{y}(t)=e(t)+n(t)$. Due to the additional last term on the right hand side of (27), the resulting error dynamics (26) will have a negative number at the $(3,3)$ entry of $\underline{A}$. This makes the adaptive synchronizer robust while under influence from external disturbances. The drawback of (27) is that even without disturbance and PE holds, synchronization errors do not converge to zero. To cope with this problem, the gain $\sigma$ in (27) can be replaced by a term proportional to the error between $\hat{y}$ and the only measurable signal $\bar{y}$, i.e.,

$$
\dot{\hat{\varphi}}=-e_{0} \Gamma \bar{\omega}(f(\bar{y}), \hat{\omega})-\kappa\left|e_{0}\right| \Gamma \hat{\varphi} \text {. }
$$

In applying the update law (28), the estimated parameters will converge to their true values when the driving signal is free of noise and the excitation is persistent.

Remark 4: In adaptive control literature [19], [20], the update laws (27) and (28) are called the $\sigma$-modification scheme and the $e_{1}$-modification scheme, respectively.

A simulation example is performed to show the effect of the modified adaptation laws. Consider the adaptive synchronization of Duffing-Holmes system same as Example 1, we then assume a noisy driving signal $\bar{y}(t)=y(t)+n(t)$ [where $n(t)$ is a white noise with zero mean and uniformly distribution and magnitude of 0.1$]$. Select the parameters $\kappa=0.0005$ in the robust update law, then the response of synchronization errors are shown in Fig. 6.

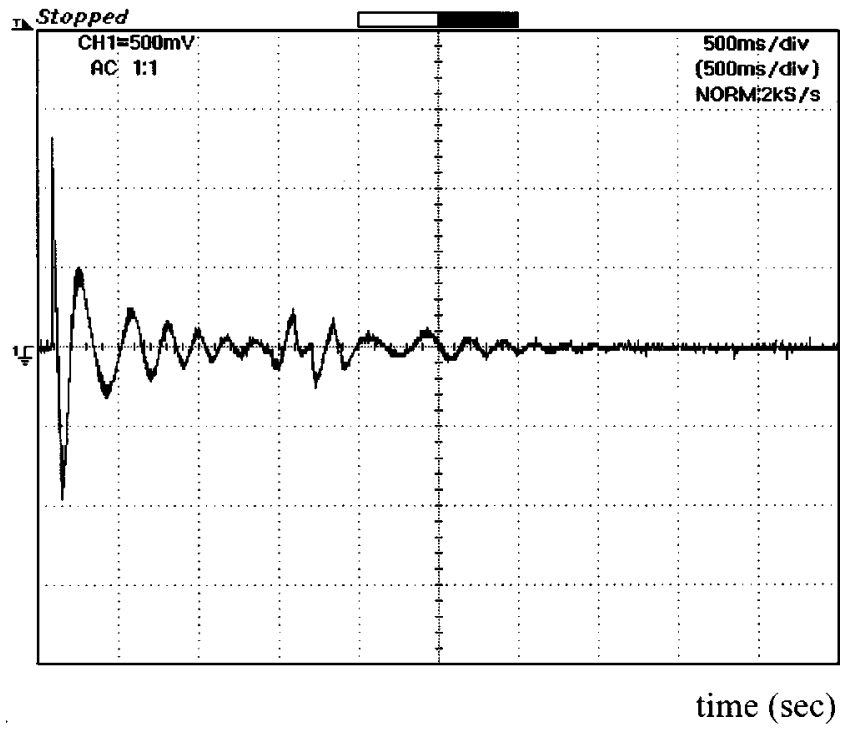

Fig. 7. Oscilloscope of DSP-based experiments of synchronization error $\hat{x}_{1}-x_{1}$ of Chua's circuit.

\section{DSP-BASED EXPERIMENTS}

To demonstrate the validity of the above-mentioned theoretical derivations, we carry out DSP-based experiments. The adaptive synchronization scheme is implemented on a dSPACE System DS1102 which has a digital signal processor (DSP) TMS320C31 on board. This system has four pairs of AD/DA channels with a 12-bit data sampling rate. Using one channel of $\mathrm{AD} / \mathrm{DA}$ as the scalar coupling signal, the remaining channels are used to output the states and/or the parameters. This setup realizes the synchronization concepts by block-diagram modeling environment. Therefore, we are able to preview the system performance prior to actual implementation. After achieving the desired results in simulated environment, the object code is generated by Real-Time Workshop and then directly downloaded into the DSP board. This process reduces the algorithm coding to a more straightforward and systematic approach, which includes coding, compiling, linking, and downloading to the target software.

The Cockpit-DSPACE software is a graphical user interface for the real-time control and fine tuning control gains online. The example used for practical implementation is the Chua circuit given in Section IV. In Figs. 7-9, the oscilloscope images of synchronization errors $\hat{x}_{1}-x_{1}$ and parameter estimation errors $\hat{a}_{3}, \hat{a}_{4}$ are shown, respectively. The results of the practical implementations are consistent to that of numerical simulations.

Potential applications can be extended from the synchronization design. For example, simple signal masking based chaotic communications; parameter division multiple access (PDMA); or synchronization based chaotic cryptosystems. The signal masking based communications, where a message is added to the output of the transmitter and then recovered at the receiver's end, provide minimal security. In PDMA, parameters of the system are modulated by binary messages [11]. The setback arises from the slow convergence of the receiver's parameters to that of the true values. To upgrade the security, standard cryptosystem theory using parameters as keys provides a more 


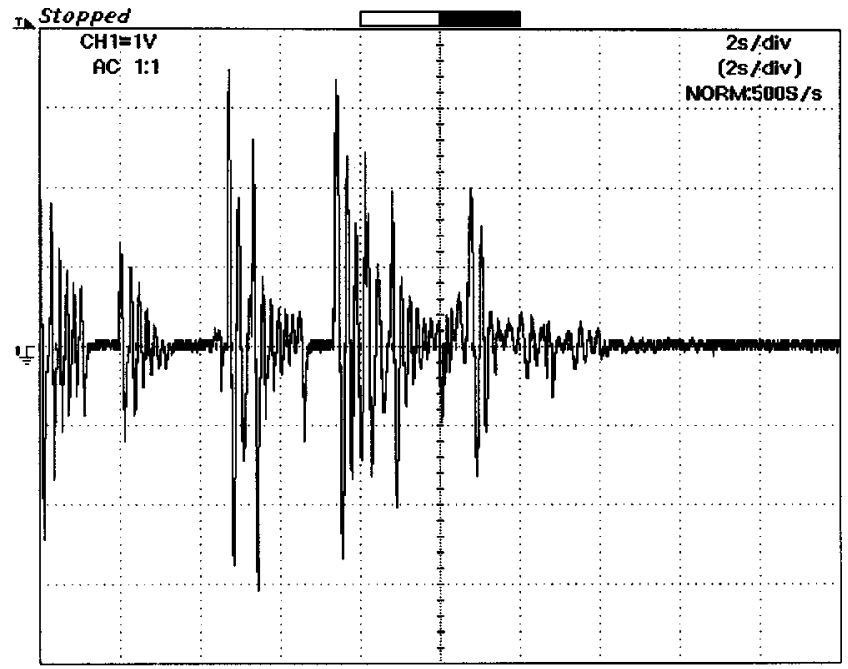

time $(\mathrm{sec})$

Fig. 8. Oscilloscope of DSP-based experiments of parameter error $\hat{a}_{3}-a_{3}$ of Chua's circuit.

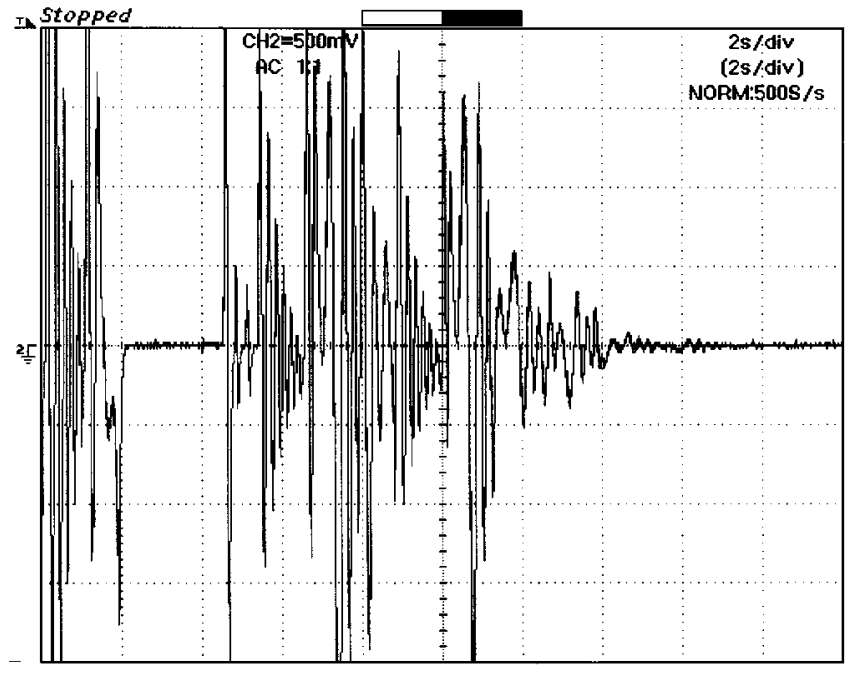

time (sec)

Fig. 9. Oscilloscope of DSP-based experiments of parameter error $\hat{a}_{4}-a_{4}$ of Chua's circuit.

secure application for chaotic synchronization based communications. Take Chua's circuit as an example, some parameters in the transmitter can be used as the key in a coding function. Then, the coding function transforms the plaintext (message) into a cyphertext. This cyphertext is then transmitted to the receiver side. Since the receiver has the same key (parameter) as the transmitter due to adaptive synchronization, we are able to decode the cyphertext. This methodology greatly enhance the security.

\section{CONCLUSIONS}

In this paper, a unified approach for synchronization and adaptive synchronization have been developed for chaotic systems of the output injection form. This approach utilizes an adaptive observer design to derive the response system for synchronization where there is parameter mismatch. Compared with other adaptive methods, this approach eliminates calculating the Lipschitz constant for nonlinearity of chaotic systems and only uses a scalar transmitted signal to drive the response system. Although the synchronization of complete states is achieved based on the convergence of estimated parameters, the condition that the injection functions must be persistent excitation can be satisfied easier for a chaotic driving signal which has a rich dynamical pattern systems. Furthermore, this method is widely applied to synchronization problems of chaotic and hyperchaotic systems after representing them in the output injection form. In presence of measurement noise, a robust adaptive response system is discussed based on the Lyapunov method. Oscilloscope images further show that for practical implementations the results are consistent with theoretical derivations.

\section{REFERENCES}

[1] L. M. Pecora and T. L. Carroll, "Synchronization in chaotic systems," Phys. Rev. Lett., vol. 64, no. 8, pp. 821-824, 1990.

[2] T. Ushio, "Synthesis of synchronized chaotic systems based on observers," Int. J. Bifurcation Chaos, vol. 9, no. 3, pp. 541-546, 1999.

[3] K.-Y. Lian, T.-S. Chiang, and P. Liu, "Discrete-time chaotic systems: Applications in secure communications," Int. J. Bifurcation Chaos, vol. 10, no. 9, pp. 2193-2206, 2000.

[4] K.-Y. Lian, T.-S. Chiang, P. Liu, and C.-S. Chiu, "LMI-based fuzzy chaotic synchronization and communication," IEEE Trans. Fuzzy Syst., vol. 9, pp. 539-553, Aug. 2001.

[5] J. Schweizer, M. P. Kennedy, M. Hasler, and H. Dedieu, "Synchronization theorem for a chaotic system," Int. J. Bifurcation Chaos, vol. 5, no. 1, pp. 297-302, 1995.

[6] H. Nijmeijer and I. M. Y. Mareels, "An observer look at synchronization,” IEEE Trans. Circuits Syst. I, vol. 44, pp. 882-890, Oct. 1997.

[7] O. Morgül and E. Solak, "Observer based synchronization of chaotic systems," Phys. Rev. E, vol. 54, no. 5, pp. 4803-4811, 1996.

[8] T.-L. Liao and N.-S. Huang, "An observer-based approach for chaotic synchronization with applications to secure communications," IEEE Trans. Circuits Syst. I, vol. 46, pp. 1144-1150, Sept. 1999.

[9] G. Grassi and S. Mascolo, "Nonlinear observer design to synchronize hyperchaotic systems via a scalar signal," IEEE Trans. Circuits Syst. I, vol. 44, pp. 1011-1014, Oct. 1997.

[10] —_, "Synchronizing hyperchaotic systems by observer design," IEEE Trans. Circuits Syst. II, vol. 46, pp. 478-483, Apr. 1999.

[11] A. Rulkov, H. Nijmeijer, and A. Markov, "Adaptive observer-based synchronization for communication," Int. J. Bifurcation Chaos, vol. 10, no. 12 , pp. 2807-2813, 2000.

[12] M. D. Bernardo, "An adaptive approach to the control and synchronization of continuous-time chaotic systems," Int. J. Bifurcation Chaos, vol. 6, no. 3, pp. 557-568, 1996.

[13] J. K. John and R. E. Amritkar, "Synchronization by feedback and adaptive control," Int. J. Bifurcation Chaos, vol. 4, no. 6, pp. 1687-1695, 1994.

[14] J. Wang and X. Wang, "Parametric adaptive control in nonlinear dynamical systems," Int. J. Bifurcation Chaos, vol. 8, no. 11, pp. 2215-2223, 1998.

[15] A. K. Kozlov, V. D. Shalfeev, and L. O. Chua, "Exact synchronization of mismatched chaotic systems," Int. J. Bifurcation Chaos, vol. 6, no. 3, pp. 569-580, 1996.

[16] C. W. Wu, Y. Tao, and L. O. Chua, "On adaptive synchronization and control of nonlinear dynamical systems," Int. J. Bifurcation Chaos, vol. 6, no. 3, pp. 455-471, 1996.

[17] A. L. Fradkov and A. Y. Markov, "Adaptive synchronization of chaotic systems based on speed gradient method and passification," IEEE Trans. Circuits Syst. I, vol. 44, pp. 905-912, Oct. 1997.

[18] A. Y. Pogromsky, "Passivity based design of synchronizing systems," Int. J. Bifurcation Chaos, vol. 8, no. 2, pp. 295-319, 1998.

[19] S. Sastry and M. Bodson, Adaptive Control: Stability, Convergence, and Robustness. Englewood Cliffs, NJ: Prentice-Hall, 1989.

[20] K. S. Narendra and A. M. Annaswamy, Stable Adaptive Systems. Englewood Cliffs, NJ: Prentice-Hall, 1989.

[21] T. Yang and L. O. Chua, "Generalized synchronization of chaos via linear transformations," Int. J. Bifurcation Chaos, vol. 9, no. 1, pp. 215-219, 1999.

[22] H. K. Khalil, Nonlinear Systems, 2nd ed. Englewood Cliffs, NJ: Prentice-Hall, 1995. 
[23] J. M. T. Thompson and H. B. Stewart, Nonlinear Dynamics and Chaos. Singapore: Wiley, 1988.

[24] C. Chen and X. Dong, From Chaos to Order Methodologies, Perspectives and Applications. Singapore: World Scientific, 1998.

[25] L. O. Chua, M. Komuro, and T. Matsumoto, "The double scroll family, Part I: Rigorous proof of chaos," IEEE Trans. on Circuits. Syst., vol. 33 , pp. 1072-1096, Nov. 1986.

[26] K. S. Narendra and A. M. Annaswamy, "A new adaptive law for robust adaptive without persistent excitation," IEEE Trans. Automat. Contr., vol. 32, pp. 134-145, Feb. 1987.

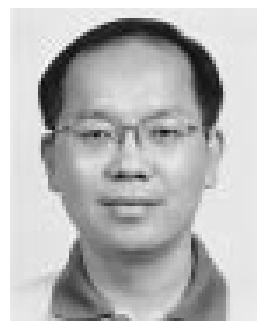

Kuang-Yow Lian (S'91-M'94) was born in Taiwan, R.O.C., in 1961. He received the B.S. degree in engineering science from National Cheng-Kung University, Taiwan, R.O.C., in 1984 , and the Ph.D. degree in electrical engineering from National Taiwan University, Taiwan, R.O.C., in 1993.

From 1986 to 1988, he served as a Control Engineer at Industrial Technology Research Institute, Taiwan. Currently, he is an Associate Professor in the Department of Electrical Engineering, Chung-Yuan Christian University, Chung-Li, Taiwan, R.O.C. His research interests include nonlinear control systems, fuzzy systems, robotics, chaotic systems, and nonholonomic control.

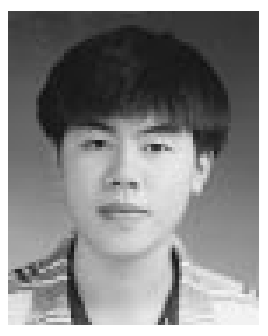

Peter Liu (S'98) received the B.S. degree in electrical engineering from Chung-Yuan Christian University, Chung-Li, Taiwan, R.O.C., in 1998, and is pursuing the Ph.D. degree in electrical engineering at the same university since 1999. His research interests include chaotic systems, nonlinear control and fuzzy systems.

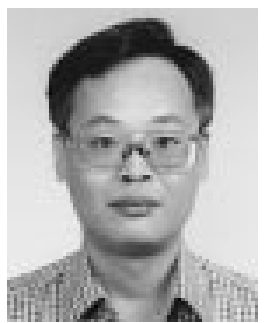

Tung-Sheng Chiang received the B.S. degree in electrical engineering and the M.S. degree in automatic control engineering, both from Feng-Chia University, Taichung, Taiwan, R.O.C., in 1984, and 1989, respectively, and the Ph.D. degree in electronic engineering, from the Chung-Yuan Christian University, Chung-Li, Taiwan, R.O.C., in 2001.

Since 1990, he has been a faculty member in the Department of Electrical Engineering, Ching-Yun Institute of Technology, Chung-Li, Taiwan, R.O.C. His research interests include nonlinear control, chaotic systems, robotics and fuzzy systems.

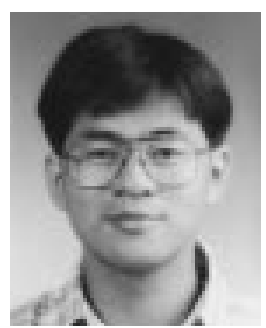

Chian-Song Chiu received the B.S. degree in electrical engineering in 1997, and the Ph.D. degree in electronic engineering in 2001, both from Chung-Yuan Christian University, Chung-Li, Taiwan, R.O.C. His research interests include robotics, fuzzy systems, and nonlinear control. 